\title{
CARACTERIZACIÓN CULTURAL Y FUNCIONAL DE ESTRUCTURAS ARQUEOLÓGICAS (EL POTRERILLO-TUCUMÁN-ARGENTINA)
}

\author{
Jimena Roldán ${ }^{(1-2-4)}$, M. Marta Sampietro Vattuone ${ }^{(2-3)}$, Liliana del V. \\ Neder ${ }^{(3)}$, Marta A. Vattuone ${ }^{(1-2)}$ y Mario Maldonado ${ }^{(3)}$. \\ (1) Insituto de Estudios Vegetales, Fac. de BQca, Qca y Fcia. (2) CONICET. (3) INGEMA, Fac. de Cs. \\ Naturales e IML. (4) Avenida Alem 114, San Miguel de Tucumán, 4000. jimena_roldan@yahoo.com
}

\section{Resumen}

Presentado el: 10/03/2009 - Aceptado 13/07/2009

El objetivo de este trabajo es realizar la caracterización cultural y funcional de las estructuras arqueológicas prehispánicas formativas de la localidad El Potrerillo (Tafídel valle-Tucumán-Argentina), a través del análisis geomorfológico del paisaje y pedogeoquímico del suelo en el cual se asentaron. Se fotointerpretó el área registrando la morfodinámica y las estructuras arqueológicas. Se excavaron siete pozos de sondeo considerando distintas situaciones contextuales, se describieron los horizontes del suelo y sus componentes y se tomaron muestras que luego fueron analizadas en laboratorio, teniendo en cuenta las concentraciones de fósforo orgánico, porcentaje de materia orgánica, macronutrientes (calcio y fósforo disponibles) y micronu trientes (hierro, cobre y manganeso disponibles). Los resultados obtenidos permitieron reconocer dos tipos de estructuras arqueológicas formativas: residenciales, evidenciadas en los restos materiales (diseños de plantas, fragmentos de cerámica, restos de carbón y cenizas) y agrícolas, por presencia de estructuras arqueológicas de terracería. La ausencia del horizonte superficial $2 \mathrm{~A}$ y de incrementos en la concentración de fósforo orgánico informan sobre la falta de prácticas conservacionistas en la actividad agrícola prehispánica del sector.

Palabras claves: Geoarqueología. Formativo. Estructuras arqueológicas. Geomorfología. Agricultura prehispánica.

\begin{abstract}
The aim of this paper was the functional and cultural characterization of formative prehispanic archaeological structures from El Potrerillo (Tafi del valle-Tucumán-Argentina) through soil pedogeochemistry and landscape geomorphological analysis. The morfodynamic and archaeological structures were registered using photo interpretation. Seven holes were dug considering different contextual situations, the soils horizons and their components were descript and samples were taken for laboratory analysis where organic phosphorous concentration, organic matter percentage, macronutrients (available phosphorous and calcium) and micronutrients (available iron, manganese and cupper) were measure. As results, two kind of formative archaeological structures were recognized: residential, shown in material objects (plant designs, pottery fragments and ashes and carbon rests) and agricultural, established by the presence of terracing archaeological structures. The lost of superficial horizon $2 \mathrm{~A}$ and the increment in organic phosphorous concentration tell us about the lack of conservational prehispanic agricultural practices in the area.
\end{abstract}

Key words: Geoarchaeology. Formative. Archaeological structures. Geomorphology. Prehispanic agricultural. 


\section{Introducción}

El hombre modifica el medioambiente que lo rodea con el fin de extraer lo que necesita para sobrevivir. Actividades como la agricultura y la ganadería fueron el pilar de la economía productiva de grupos humanos prehispánicos formativos en el valle de Tafí. Esta estructuración social trajo aparejada una conducta sedentaria evidenciada por la construcción de unidades residenciales permanentes.

Para poder entender la relación que se estableció entre los grupos humanos pasados y el medioambiente que habitaron utilizamos métodos y técnicas provenientes de las ciencias geológicas. La disciplina que nace a partir de este nuevo enfoque es conocida como geoarqueología.

Los componentes primarios de su estudio son la reconstrucción del contexto paisajístico y estratigráfico, la valoración de los procesos de formación que intervinieron en un yacimiento tanto de índole natural como cultural, la valoración de los procesos que modificaron el yacimiento y la identificación de los rasgos de actividad humana sobre el paisaje considerando al hombre como agente geomórfico, que transforma el paisaje introduciendo y modificando materiales orgánicos e inorgánicos. Estos materiales están expuestos a una constante fragmentación y degradación mecánica y química. La utilización, reutilización y eliminación de estos materiales producen alteraciones en el perfil sedimentológico (Butzer 1989), que pueden ser medidas a través del análisis pedogeoquímico.

Para este trabajo partimos de dos principios teóricos. Por un lado, cada unidad geomorfológica es una unidad ambiental básica, adecuada para discriminar cualidades y categorías de paisaje, que posee génesis y evolución temporal comunes a toda su superficie y homogeneidad espacial dada por la recurrencia de elementos morfogenéticos endógenos (Sayago y Collantes 1991).

Este concepto permite delimitar un área de trabajo en la cual el desarrollo natural del suelo y el paisaje fue relativamente uniforme, implicando que las alteraciones observadas serían producto de la actividad humana.

Por el otro, se definen a los suelos antrópicos como artefactos arqueológicos susceptibles de ser estudiados como tales. Pueden caracterizarse tipológicamente y brindan información sobre las relaciones espaciales y el modo de vida de las poblaciones que habitaron previamente una parcela de suelo dada (Sampietro Vattuone 2007).

Basándonos en este principio sabemos que la actividad humana deja improntas en el suelo que son susceptibles de ser estudiadas a través del análisis de concentraciones diferenciales de elementos y compuestos químicos, como así también de su distribución vertical (en un perfil) y horizontal (en una geoforma o superficie habitacional).

Bajo estas premisas, el objetivo de este trabajo es realizar la caracterización cultural y funcional de las estructuras arqueológicas prehispánicas formativas del área de estudio, a través del análisis geomorfológico del paisaje y pedogeoquímico del suelo en el cual se asentaron. 


\section{Área de estudio}

El área de estudio se ubica en el piedemonte del Cerro Nuñ̃orco Grande, en la zona de El Potrerillo y en el cerro Loma Pelada (El Mollar, valle de Tafí), la vía de acceso a la misma es la ruta provincial 355, la cual desciende hacia el sur por la Quebrada del Portugués. El piedemonte está formado por una unidad geomorfológica de origen denudativo denominada glacis cubierto. Estas son formas pedemontanas relativamente planas a onduladas, de morfometría similar a los glacis de erosión, pero caracterizadas por una cubierta clástica de aproximadamente $15 \mathrm{~m}$ de espesor, que cubre los materiales loéssicos finipleistocenos de la Formación Tafí del Valle subyacentes (Collantes 2001). Figura 1.

\section{Arqueología del valle de Tafí}

El valle de Tafí fue el asiento de la cultura formativa Tafí. Su ocupación se extendió desde el año 94 al 1.001 d.C. (González y Núñez Regueiro 1960 y Berberián et al. 1988). Se identificaron tres tipos de sistemas estructurales: agrícola-ganadero, residencial y ceremonial (Berberián et al. 1988, González y Nuñez Regueiro 1960 y Nuñez Regueiro y Azcárate 1996).

Esta entidad sociocultural fue caracterizada a partir de su escultórica lítica, representada por los menhires y máscaras de piedra; patrón de asentamiento compuesto por estructuras circulares de piedra aisladas o adosadas a un patio central, en un número variable, que ocasionalmente formaban conjuntos más complejos; estructuras agrícolas, que comprendían canchones y/o andenes de cultivo; y un centro ceremonial constituido por un montículo asociado a menhires en el sitio Casas Viejas (El Mollar) (Berberián et al. 1988, Sampietro Vattuone 1994, 2002 y Tartusi y Nuñez Regueiro 1993).

Ésta cultura abarca un lapso temporal prolongado (Berberián et al. 1988, González y Nuñez Regueiro 1960, González 1965 y Sampietro Vattuone 1999), lo que llevó a cambios tecnológicos y económicos durante su existencia, por ello se la dividió en dos etapas culturales denominadas Tafí I o La Angostura (siglos I al IV d.C.) y Tafí II o Carapunco (siglos VII al X d.C.) (Nuñez Regueiro y Azcárate 1996).

Los recintos tienen aspecto predominantemente circular, pueden formar estructuras complejas, compuestas, dobles o simples. Las estructuras residenciales o domésticas se componen de un círculo central mayor (10 a $20 \mathrm{~m}$ de diámetro) rodeado de círculos más pequeños ( 2 a $6 \mathrm{~m}$ de diámetro), en algunos casos se encuentran vinculadas a terrazas de cultivo. Dentro de este sistema, también se incluyen plantas de tipo cuadrangular y montículos (Sampietro Vattuone 1994).

Por otro lado, la arquitectura agrícola está representada por líneas de despedre, andenes de cultivo, canchones de cultivo y canales de irrigación (Sampietro Vattuone 1994).

La distribución de asentamiento encontrados en el valle esta íntimamente relacionada con las diferentes unidades geomorfológicas identificadas, donde los conos glacis y los abanicos aluviales fueron utilizados para el asiento de estructuras tanto agrícolas como residenciales; en los glacis cubiertos prevalecieron las estructuras residenciales; y en los glacis de erosión se observaron algunas unidades residenciales dispersas y círculos aislados (Sampietro Vattuone 2002). 


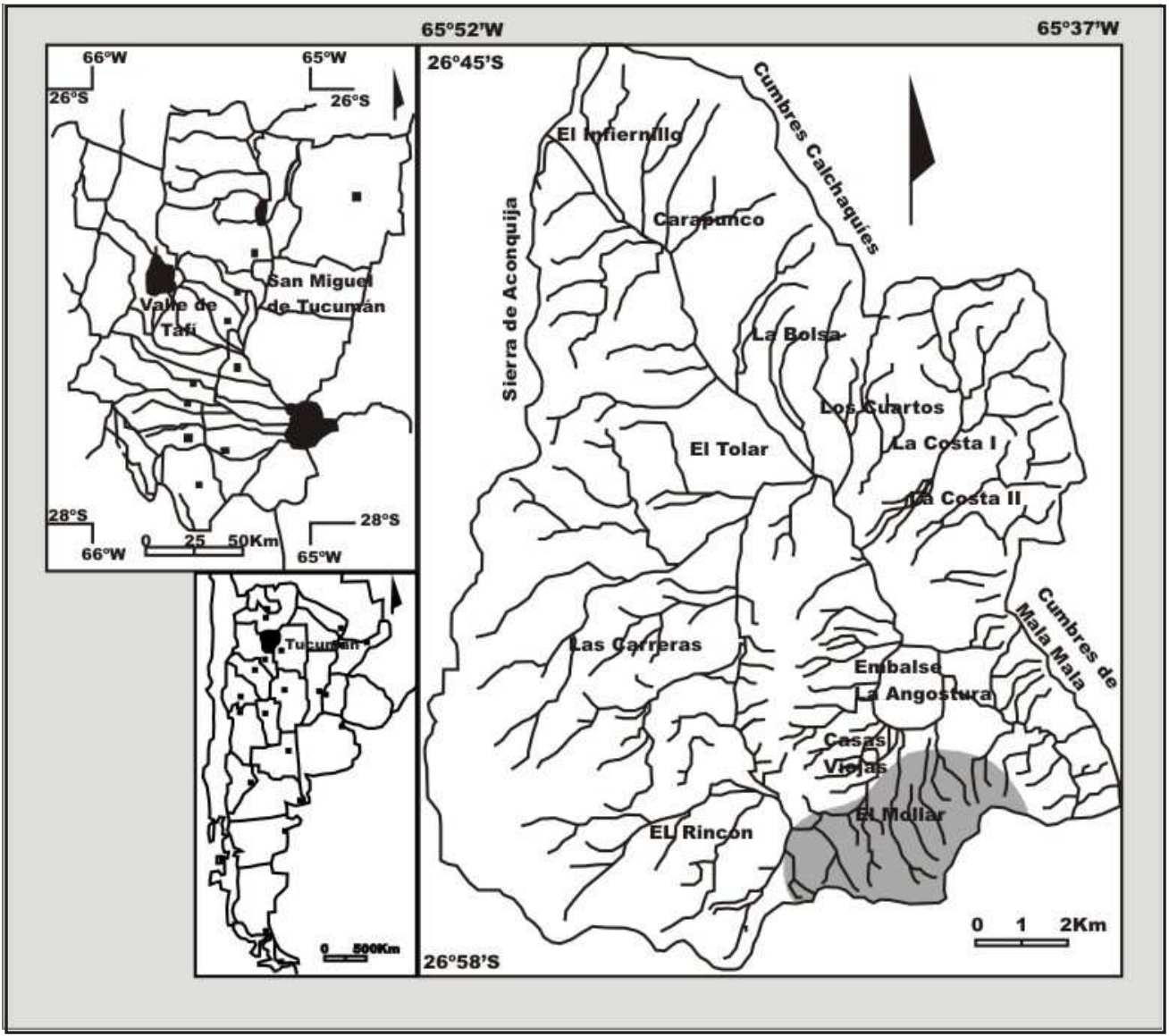

Figura 1. Mapa de ubicación. El área de estudio está resaltada en gris.

Un estudio detallado (análisis pedogeoquímicos) en los suelos de las estructuras agrícolas encontradas en el galcis cubierto de El Tolar (Tafí del Valle) brindó información relacionada con un óptimo desarrollo de los suelos para la actividad agrícola, y una elevada probabilidad de haber recibido aportes externos de nutrientes a través de la utilización de fertilizantes orgánicos (Roldán et al. 2008)

\section{Métodos y técnicas}

Mediante fotointerpretación (escala 1:20.000) se mapearon las unidades morfogenéticas y la distribución de estructuras arqueológicas correspondientes al Período Formativo.

Las fotos aéreas fueron georreferenciadas y la información obtenida fue digitalizada con tableta digitalizadora o directamente por interpretación visual desde pantalla, utilizando el programa ILWIS 3.3 para la elaboración de la cartografía temática y la integración de los resultados. La misma fue controlada a través de prospecciones pedestres. 
Para recopilar la información pedológica asociada a las actividades antrópicas pasadas se realizaron siete sondeos en diversos contextos arqueológicos. Tras efectuar la descripción de acuerdo a las normas propuestas por Etchevere (1976), se muestreó el sector medio de cada horizonte identificado.

Las muestras fueron analizadas en laboratorio considerando el porcentaje de materia orgánica y las concentraciones de micronutrientes (hierro, cobre y manganeso disponibles) y macronutrientes (calcio y fósforo disponibles), dado su valor diagnóstico y por tratarse de elementos activos en los procesos geoquímicos y bioarqueológicos (Buckman y Brady 1977).

Con el objeto de tener un indicador de actividad humana y sus características, se determinó la concentración de fósforo orgánico, dada su capacidad para mantenerse estable por largos períodos de tiempo (Terry et al. 2000).

El fósforo disponible fue determinado a partir del método de azul de molibdeno. Para fósforo total se aplicó este mismo método, previa digestión de la muestra con ácido sulfúrico. El fósforo orgánico fue estimado por diferencia entre ambos (Roldán et al. 2005).

El calcio se determinó por el método complexiométrico utilizando EDTA Na2 y murexida (Roldán et al. 2005).

El hierro disponible fue determinado extractándolo con acetato amónico-ácido acético y por reducción con clorohidrato de hidroxilamina, posteriormente se leyó la absorbancia en espectrofotómetro a 508 milimicrones de longitud de onda. El cobre disponible se determinó utilizando el mismo extractante que para hierro, luego de tratarlo con EDTA $\mathrm{Na} 2$ y citrato amónico fue titulado con rojo cresol y solución de amoníaco. Para separar la fase orgánica y eliminarla, se añadieron dietilditiocarbamato sódico y tetracloruro de carbonato. Luego se leyó a 440 milimicrones de longitud de onda. Finalmente, el manganeso disponible fue tratado con acetato amónico neutro y luego de oxidar la materia orgánica con peróxido de hidrógeno y eliminar este último, se leyó la absorbancia con espectrofotómetro a 540 milimicrones de longitud de onda (Roldán et al. 2005).

El porcentaje de materia orgánica fue obtenido utilizando el método de Walkley y Black (Roldán et al., 2005).

\section{Resultados}

\section{El paisaje}

El área de estudio está compuesta por la ladera sur de la Loma Pelada y un glacis cubierto (Collantes 2001) que ocupa gran parte del piedemonte del cerro Ñuñorco Grande.

A partir de estudios morfodinámicos se observa un predomino de procesos de erosión hídrica de grado severo, dando como resultado la formación de barrancos en forma de $\mathrm{V}$, U y 
fondo plano, escarpas de erosión por remoción lateral de cauce incipiente y cárcavas incipientes y ramificadas con retroceso de cabeceras. Figura 2.

A esta dinámica natural hay que sumarle la acción antrópica que provoca la aceleración de estos procesos. El desarrollo urbano, el sobrepastoreo y las prácticas agrícolas no conservacionistas son algunos de los factores responsables.

\section{Patrón de distribución de rasgos arquitectónicos prehispánicos}

A partir de la fotointerpretación pudieron identificarse estructuras circulares (de 2 a $18 \mathrm{~m}$ de diámetro) y rectangulares de dimensiones variables (desde $4 \times 5 \mathrm{~m}$ a $23 \times 18 \mathrm{~m}$ ). Se observan formas conocidas de estructuras circulares aisladas (estructuras simples), dos o más estructuras circulares de similar tamaño adosadas unas a otras (estructuras dobles), estructuras circulares grandes que tienen adosadas estructuras circulares más pequeñas (estructuras compuestas) y dos o más estructuras como la descripta previamente adosadas entre sí (estructuras complejas).

Entre los complejos estructurales no descriptos hasta ahora se observan de 5 a 10 estructuras circulares de 2,5 a $6 \mathrm{~m}$ de diámetro adosadas, formando una cadena que se extiende en línea recta, perpendicular a la pendiente. Estructuras circulares, de dimensiones variables, parcialmente superpuestas unas con otras, dando la impresión de que su construcción y utilización se llevó a cabo en momentos diferentes, ya que una de ellas por lo general tiende a encontrarse mejor conservada que la subyacente.

Entre las estructuras rectangulares se encontraron patrones ya definidos, como estructuras rectangulares aisladas de diferentes tamaños y dos o más estructuras de dimensiones similares adosadas entre sí. Figura 3.

También se observaron combinaciones de estructuras circulares y rectangulares: (a) estructuras rectangulares que tienen adosadas una o más estructuras circulares pequeñas; $y$ (b) estructuras rectangulares que en su interior albergan una estructura circular pequeña (hasta la fecha este tipo de plantas no han sido asociadas al patrón formativo por lo que en esta investigación no fueron analizadas). Figura 3.

Las estructuras agrícolas son las más difíciles de identificar debido a sus formas poco convencionales, a la existencia de construcciones urbanas, como así también a los accidentes del terreno que, en muchos casos, simulan terrazas. A pesar de ello, fue posible distinguir algunos andenes y líneas de despedre que tenían adosadas, en algunos casos, estructuras circulares pequeñas de no más de $2 \mathrm{~m}$ de diámetro. Figura 3.

En la zona se registraron estructuras rectangulares que probablemente hayan funcionado como canchones de cultivo, ya que superficialmente son de tamaño considerable $(26,5 \times 16,2$ $\mathrm{m})$, están adosadas entre sí y los muros paralelos a la pendiente son sinuosos mostrando características propias a las llamadas "líneas de despedre" por Sampietro Vattuone (1994). Figura 3. 


\section{Física y química de suelos}

De acuerdo a las características observadas en las descripciones de campo se pueden establecer dos secuencias pedológicas: un suelo antiguo o paleosuelo reconocido por diferencias de color, tipo de estructuras y texturas y el suelo actual. En éste último la secuencia de horizontes se caracteriza por la presencia de un $\mathrm{A}$, seguido de un $\mathrm{BC}, \mathrm{AB}$ o $\mathrm{AC}$ poco desarrollados y en algunos casos pudo identificarse el C.

Las descripciones del paleosuelo muestran la pérdida del horizonte superficial 2A en todos los perfiles (con excepción del perfil 5). En términos generales se encuentra a partir de los $25 \mathrm{~cm}$ de profundidad y los horizontes iluviales están bien desarrollados.

El límite inferior de los perfiles está determinado por un flujo arenoso de forma ondulada, apareciendo entre los 34 y $59 \mathrm{~cm}$ de profundidad.

La presencia de una capa de sedimento compactado, restos arqueológicos (alfarería, cenizas y carbón) y el límite suelo/paleosuelo en el perfil 5, informan que el nivel de ocupación prehispánico es contemporáneo al suelo enterrado. Cuadro 1.

El análisis pedogeoquímico realizado en laboratorio da cuenta de concentraciones de fósforo orgánico relativamente uniformes a lo largo de los perfiles que abarcan valores de $2.639 \mathrm{ppm}$ y $1.522 \mathrm{ppm}$. Por otro lado, los porcentajes de materia orgánica tienden a ser bajos y a decrecer con la profundidad (desde $0,35 \%$ a $0,75 \%$ ). Cuadro 1.

Dentro de los macronutrientes, el fósforo disponible tiende a aumentar con la profundidad, el rango de concentración varía entre 87 ppm y 189 ppm. La dinámica del calcio disponible es más compleja, debido a su capacidad para moverse verticalmente en el perfil, tiende a acumularse en los horizontes iluviales con un valor promedio de $1,4 \times 106 \mathrm{ppm}$, mientras que en el resto de los horizontes la concentración promedio es de 1,2 x $106 \mathrm{ppm}$. Cuadro 1.

En cuanto a los micronutrientes biodisponibles considerados, en el perfil 1 las concentraciones de hierro y manganeso se mantienen relativamente constantes, mientras que el cobre disminuye considerablemente con la profundidad (de 19,35 ppm a 0,61 ppm). En el perfil 4 los tres elementos aumentan con la profundidad, siendo este incremento en la concentración más significativo en el cobre (de 2,86 ppm a 8,87 ppm). En el caso del perfil 5, teniendo siempre en cuenta su singularidad, en el primer paleosuelo los valores de hierro aumentan en profundidad (de $0,8 \mathrm{ppm}$ a $4,3 \mathrm{ppm}$ ) mientras que los de cobre disminuyen (de $10,33 \mathrm{ppm}$ a $0,53 \mathrm{ppm}$ ) y los de manganeso se mantienen relativamente constantes (de 1,7 ppm a 1,1). Paralelamente, en el segundo paleosuelo los valores de hierro disminuyen con la profundidad (de 2,3 ppm a 0 ppm), los de cobre aumentan (de 0,39 ppm a 15,13 ppm) y los de manganeso se mantienen constantes $(0,8 \mathrm{ppm}$ a $0,6 \mathrm{ppm})$. Por último, en el perfil 6 , la concentración de manganeso es constante a lo largo del perfil (1,1 ppm a 1,2 ppm), el cobre disminuye con la profundidad (6,01 ppm a 2,96 ppm) y no se encontró hierro. Cuadro 1. 


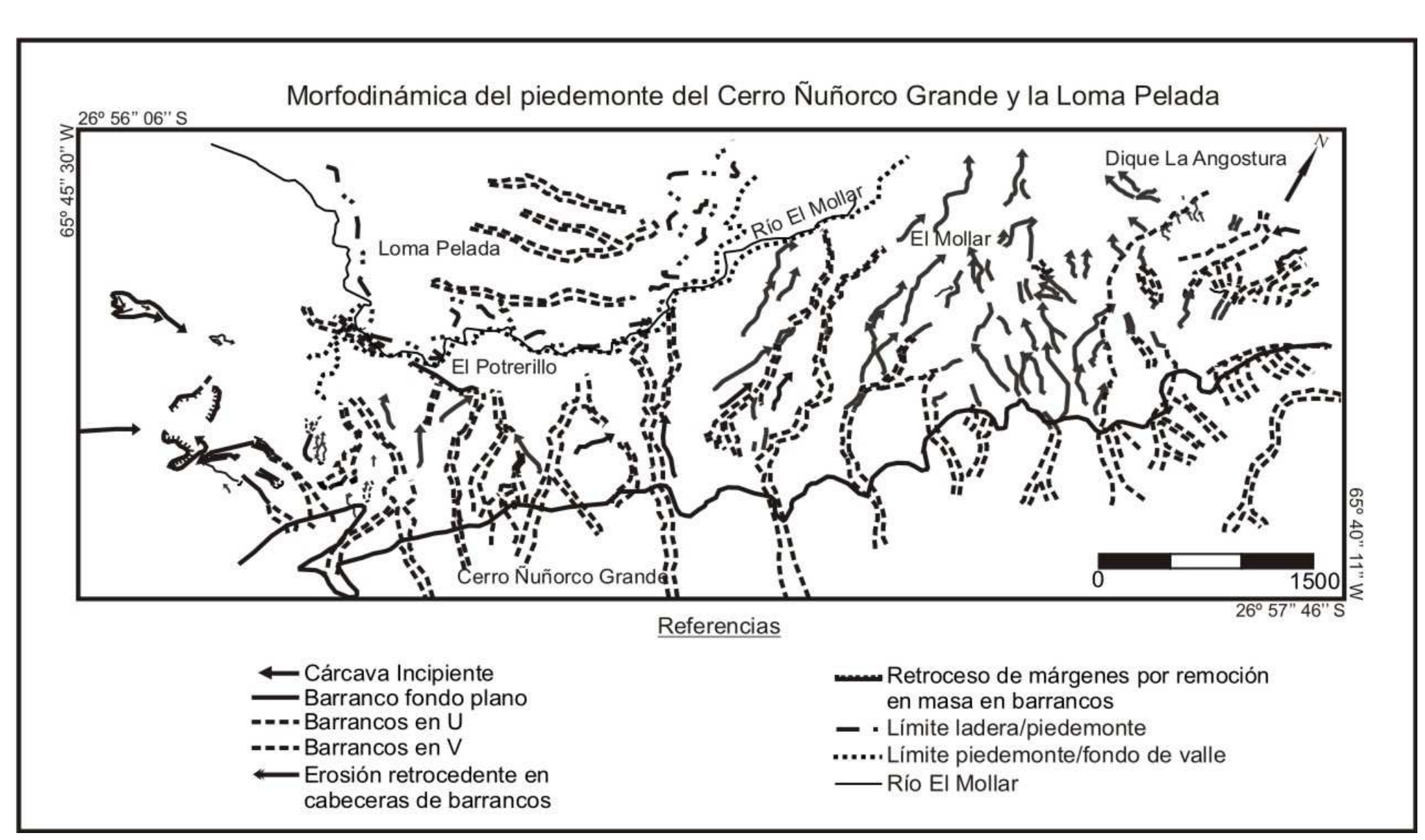

Figura 2. Mapa morfodinámico del piedemonte del cerro Ñuñorco Grande. El Mollar, valle de Tafí. 


\section{Discusión}

La conservación y visibilidad actual de las unidades arquitectónicas de pertenencia prehispánica formativa responden a la dinámica de las unidades de paisaje que componen el área de estudio. Los procesos erosivos intensivos, productos de las actividades natural y antrópica, reflejados en la presencia de cárcavas y barrancos como resultado de la erosión hídrica, la agricultura sin prácticas conservacionistas, el sobrepastoreo y la urbanización afectaron el nivel de conservación de las diferentes estructuras arqueológicas reconocidas. Figura 2.

Estas actividades modificaron el paisaje arquitectónico del formativo haciendo muy difícil la reconstrucción, hoy en día, del patrón de distribución de las estructuras que lo componen. Figura 3.

Para poder identificar las actividades practicadas en ellas se realizaron una serie de muestreos dentro de las estructuras ubicadas en el glacis cubierto. Su análisis muestra suelos que responden a características ambientales propias de la zona, donde la composición del material parental, la topografía (pendiente pronunciada de corta longitud), las características texturales (gruesas y permeables), los procesos de erosión hídrica (particularmente la mantiforme) y la acción antrópica actual (pastoreo, agricultura y urbanización) dieron lugar a la evolución de suelos permeables y poco desarrollados, donde se observa cierta uniformidad en la concentración de los elementos analizados. Cuadro 1.

En la descripción de los perfiles se encontró un suelo enterrado identificado como el nivel de ocupación prehispánico. La evidencia que respalda esta afirmación provino de los restos materiales y de los resultados obtenidos a partir del análisis físico-químico de los horizontes del perfil 5. En éste, el piso de ocupación (3A), muy compactado, se ubicaba a unos $55 \mathrm{~cm}$ de profundidad, sobre él se hallaron fragmentos de cerámica tosca y restos de carbón y cenizas. Por encima del mismo se depositó una capa de sedimento arenoso (2C2) como resultado de su abandono. Posteriormente colapsó el techo y sobre este se formó un suelo (2B) cuyo desarrollo se vio interrumpido por la depositación de otra capa sedimentaria, a partir de la cual evolucionó el suelo actual. Cuadro 1.

Los procesos postdepositacionales que se desarrollaron dentro de dicha estructura provocaron la formación de un segundo suelo enterrado (vinculado al colapso del techo prehispánico), situación no registrada en el resto de los perfiles.

La fotointerpretación y la prospección pedestre de dicha estructura la definían como de tipo agrícola. Sin embargo, los restos materiales recuperados, junto con los resultados de laboratorio, indican actividades propias de una unidad habitacional. La dinámica pedológica muestra un horizonte superficial 3A intacto en el nivel de ocupación, situación ya observada en otras regiones del valle de Tafí (Roldán et al. 2008).

Se encontraron andenes de cultivo dentro del glacis cubierto, situación contraria a la que Sampietro Vattuone (2002) describe para el glacis cubierto del piedemonte de cumbres Calchaquíes, dentro del mismo valle. En este caso, es probable que la diferencia altitudinal entre ambas geoformas (3.000 msnm a la altura de El Infiernillo y 1.980-2.320 msnm en el piedemonte del cerro Nuñorco Grande), sumado a las corrientes de vientos del valle haya 


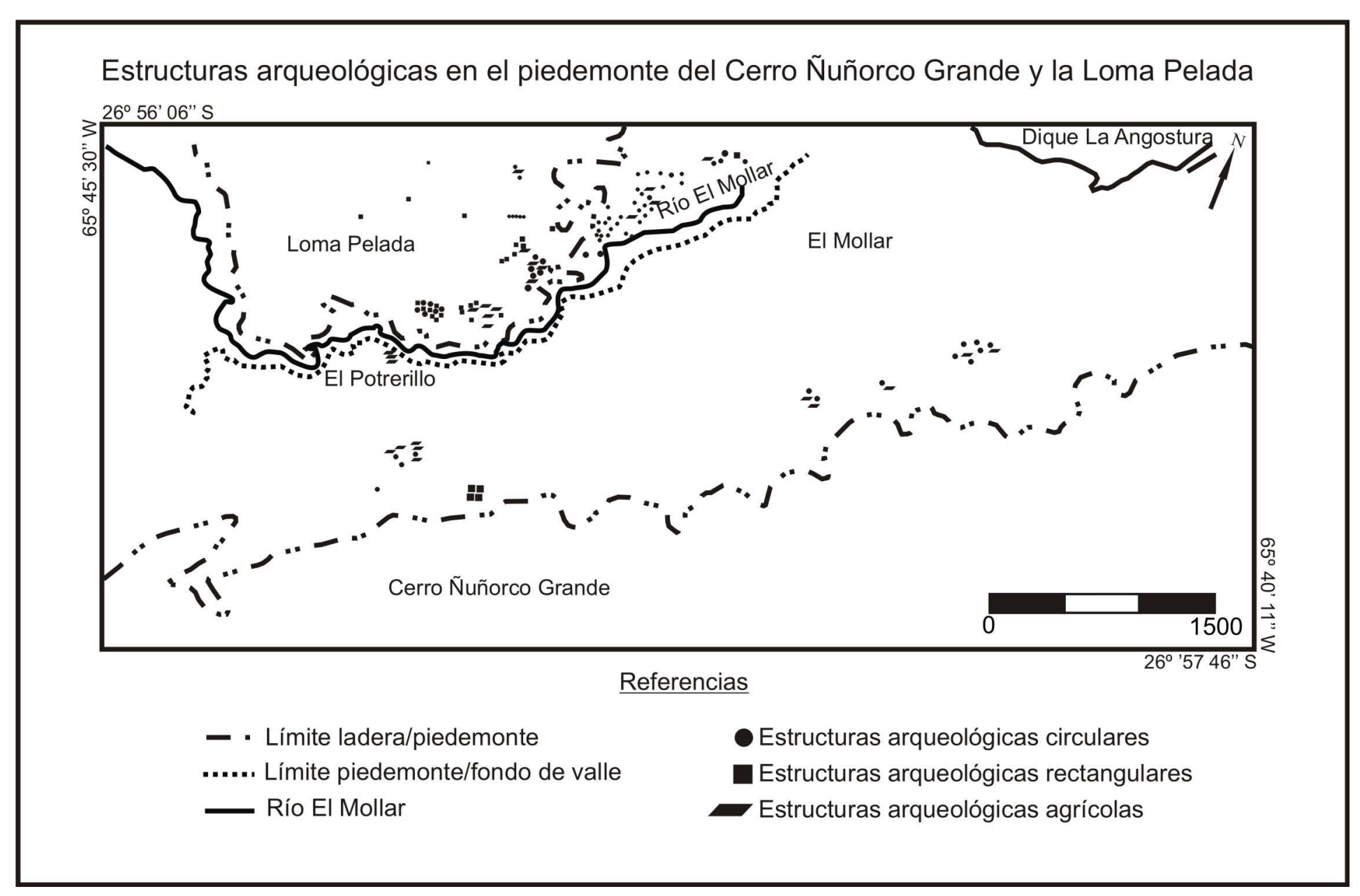

Figura 3. Mapa de distribución de estructuras arqueológicas. El Mollar, valle de Tafí 
significado mayor resguardo de los vientos fríos del sur para este sector, facilitando la actividad agrícola.

Los análisis de laboratorio muestran un suelo pobre en elementos necesarios para el buen desarrollo de plantas cultivadas, hecho manifiesto en porcentajes bajos de materia orgánica, estructuras poco desarrolladas, escasa potencia de los horizontes y la dinámica descrita en la concentración de los micronutrientes. Cuadro 1.

Por otro lado, las concentraciones de fósforo orgánico no registran valores indicativos de actividad humana. Esto se debe posiblemente a la degradación de los suelos, donde el horizonte superficial fue erosionado y por ende el elemento en cuestión, y/o a la falta de fertilización de los suelos bajo actividad agrícola. Situaciones similares fueron observadas por Sandor et al. (1986) en suelos sometidos a actividad agrícola en una región semiárida de Nuevo México. Sin embargo, resultados previos obtenidos en otro sector del valle muestran un importante incremento de este elemento en el nivel de ocupación prehispánico (Roldan et al. 2008).

Paralelamente, la baja concentración de materia orgánica provocó cambios significativos en las concentraciones de micronutrientes (cobre, hierro y manganeso disponibles) y en la relación entre ellos. El porcentaje de materia orgánica es un factor determinante en la presencia, distribución y biodisponibilidad de los micronutrientes del suelo (Roca et al. 2007, Reichaman 2002).

Las concentraciones de Mn disponible aumentan con la profundidad, situación inversa a la observada cuando los porcentajes de materia orgánica permiten su asociación, lo cual provoca que los mayores valores de manganeso se encuentren en superficie, por su fuerte afinidad a los complejos órgano-minerales que evitan su pérdida por lixiviación (Roca et al. 2007).

La estabilidad de los complejos de cambio y quelatos (que son el producto de la reacción de la materia orgánica con los metales) depende del porcentaje de materia orgánica distribuido en los horizontes superficiales de un suelo, donde el cobre es el elemento más fuertemente adsorbido por la materia orgánica, no quedando disponible para las plantas (Bohn et al. 1993) y el manganeso es asimilado en cantidades menores. Por ende, la biodisponibilidad del manganeso es mayor, seguido por el hierro y finalmente el cobre. Sin embargo, cuando el porcentaje de materia orgánica no es suficientemente importante como para adsorber micronutrientes esta relación no se cumple y obtenemos concentraciones de cobre mayores que de manganeso, sin que los mismos lleguen a ser fitotóxicos.

Por lo tanto, los resultados obtenidos para los perfiles mencionados no invalidan la posibilidad de un uso agrícola prehispánico de estos suelos, lo que si podemos decir es que las bajas concentraciones de fósforo orgánico indican la ausencia de fertilizantes para la manutención de los suelos bajo cultivo, situación respaldada por el comportamiento observado para la distribución de materia orgánica y micronutrientes disponibles.

\section{Conclusiones}

La dinámica natural y antrópica sufridas por el paisaje donde se asientan las estructuras arqueológicas estudiadas provocaron cambios en las formas y en el grado de visibilidad actual de las mismas. 


\begin{tabular}{|c|c|c|c|c|c|c|c|c|c|c|c|c|c|c|}
\hline 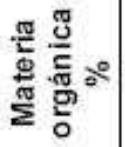 & $\stackrel{\mathscr{E}}{=}$ & $\stackrel{\mathscr{N}}{=}$ & $\begin{array}{l}\text { की } \\
0 \\
0\end{array}$ & $\begin{array}{l}8 \\
0 \\
0\end{array}$ & ơ & $\begin{array}{l}\stackrel{D}{\infty} \\
\sim \\
\sim\end{array}$ & $\stackrel{\mathscr{E}}{-}$ & $\stackrel{\infty}{\circ}$ & ले & $\stackrel{\substack{m \\
0}}{0}$ & $\begin{array}{l}F \\
\text { N }\end{array}$ & $\stackrel{\infty}{\stackrel{\circ}{-}}$ & - & 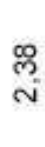 \\
\hline 웡 & $\begin{array}{l}\mathbb{0} \\
\infty \\
\infty\end{array}$ & $\begin{array}{l}\bar{m} \\
\text { চ্口 }\end{array}$ & $\begin{array}{l}\bar{n} \\
\text { 心 }\end{array}$ & $\begin{array}{l}\text { ల్ } \\
\text { Ф }\end{array}$ & $\bar{\emptyset}$ & 仓े & लొ & 㣻 & 芯 & $\stackrel{\infty}{\sim}$ & స్ స & $\begin{array}{l}R \\
\text { N }\end{array}$ & $\begin{array}{l}\mathscr{8} \\
\sim \\
\sim\end{array}$ & $\approx$ \\
\hline 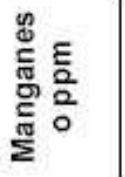 & $\hat{0}$ & $\stackrel{m}{=}$ & $\stackrel{m}{=}$ & $\hat{0}$ & $\hat{o}$ & $\stackrel{\circ}{-}$ & $\stackrel{\Delta}{-}$ & : & $\ddot{0}_{0}^{\prime}$ & $\tilde{O}_{0}^{N}$ & $\stackrel{\infty}{=}$ & $\stackrel{\sim}{=}$ & a) & $\stackrel{\square}{-}$ \\
\hline 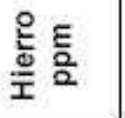 & $\stackrel{5}{\infty}$ & $N$ & 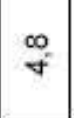 & in & iี & $\overline{5}$ & $\stackrel{\infty}{\stackrel{\infty}{m}}$ & $\begin{array}{l}\infty \\
\mu^{\infty} \\
0\end{array}$ & $\bar{N}$ & $\begin{array}{l}\mathscr{N} \\
\mathbb{N}\end{array}$ & $\bar{\Gamma}$ & $\stackrel{\ddot{N}}{\sim}$ & $\stackrel{\infty}{\sim}$ & $\stackrel{N}{N}$ \\
\hline$\frac{ㅇ ㅡ ㅇ ~}{\frac{u}{\pi}} \frac{\mathrm{g}}{2}$ & $\begin{array}{l}8 \\
\delta \\
8 \\
0\end{array}$ & $\begin{array}{l}\mathscr{e} \\
\stackrel{0}{0} \\
0 \\
\text { m }\end{array}$ & $\begin{array}{l}\stackrel{8}{0} \\
0 \\
\stackrel{0}{\circ} \\
=\end{array}$ & $\begin{array}{l}\mathscr{8} \\
\stackrel{ल}{\circ} \\
\stackrel{\rho}{2}\end{array}$ & $\begin{array}{l}\text { \%ั } \\
\text { \% } \\
\text { \% } \\
\end{array}$ & $\begin{array}{l}\text { 요 } \\
\infty \\
\mathbb{\infty} \\
\infty \\
\infty\end{array}$ & 志 & 怘 & 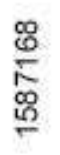 & $\begin{array}{l}\text { 워 } \\
\text { స్ల్ } \\
\text { 인 }\end{array}$ & 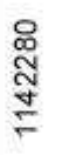 & 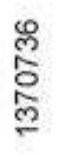 & 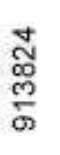 & $\begin{array}{l}0 \\
\stackrel{0}{o} \\
\text { \& } \\
\infty \\
\infty\end{array}$ \\
\hline 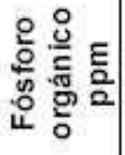 & $\begin{array}{l}\mathscr{Q} \\
\stackrel{6}{6}\end{array}$ & 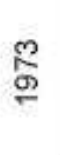 & $\stackrel{N}{\stackrel{N}{N}}$ & $\frac{8}{\sim}$ & $\stackrel{\text { J }}{\stackrel{N}{N}}$ & $\begin{array}{l}\text { : } \\
\text { ్ㅗ }\end{array}$ & $\begin{array}{l}0 \\
\mathbb{6} \\
5\end{array}$ & §్ & $\begin{array}{l}\stackrel{ळ}{œ} \\
\stackrel{\circ}{\rightleftharpoons}\end{array}$ & $\begin{array}{l}\text { ల్లి } \\
\text { స్ }\end{array}$ & $\frac{\mathfrak{d}}{\mathrm{d}}$ & \begin{tabular}{l}
$\mathscr{0}$ \\
\hdashline
\end{tabular} & $\begin{array}{l}\stackrel{0}{8} \\
=\end{array}$ & $\underset{2}{\stackrel{2}{\sigma}}$ \\
\hline 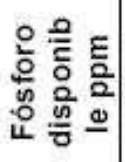 & ్ㅏㄹ & $\bar{N}$ & $\stackrel{ }{\Re}$ & $\cong$ & 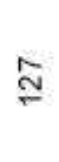 & ஜू & 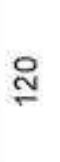 & $\stackrel{\infty}{\circ}$ & $\stackrel{0}{ \pm}$ & $\stackrel{\mathscr{0}}{\infty}$ & 언 & 吕 & $\stackrel{\infty}{\stackrel{\sim}{\simeq}}$ & 음 \\
\hline 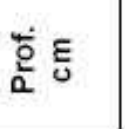 & İ & $\frac{\sigma}{\frac{\pi}{4}}$ & \begin{tabular}{l}
\multirow{2}{*}{} \\
Oे
\end{tabular} & $\stackrel{\stackrel{N}{N}}{\sim}$ & $\begin{array}{l}\text { के } \\
6 \\
\text { ొొ }\end{array}$ & पे & $\frac{\varphi}{7}$ & $\begin{array}{l}\stackrel{?}{?} \\
\stackrel{0}{2}\end{array}$ & $\begin{array}{l}\text { ปे } \\
\text { है }\end{array}$ & $\begin{array}{l}0 \\
\text { o } \\
\text { bे }\end{array}$ & $\frac{\dot{d}}{d}$ & 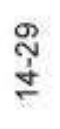 & $\begin{array}{l}\text { ભे } \\
\text { के }\end{array}$ & $\bar{\delta}$ \\
\hline 荧 & $\ll$ & ర్ల & 0 & 迎 & $\stackrel{\mathscr{\sim}}{\sim}$ & $\ll$ & $\stackrel{m}{\varangle}$ & 0 & 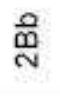 & $\stackrel{m}{\sim}$ & $\ll$ & U & 0 & $\varangle$ \\
\hline & \multicolumn{5}{|c|}{ Perfil 1} & \multicolumn{6}{|c|}{ Perfil 2} & \multicolumn{2}{|c|}{ Perfil 3} & \\
\hline
\end{tabular}

Cuadro 1. Resultados de laboratorio de las muestras de suelo extraidas del glacis cubierto. El Mollar, valle de Tafí. 


\begin{tabular}{|c|c|c|c|c|c|c|c|c|c|c|c|c|c|c|}
\hline 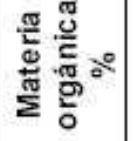 & $\begin{array}{l}\infty \\
\infty \\
\infty \\
-\end{array}$ & $\stackrel{m}{\sim}$ & $\stackrel{\mathscr{2}}{\stackrel{2}{0}}$ & $\begin{array}{l}\text { ¿ } \\
\stackrel{\text { ले }}{ }\end{array}$ & Б) & $\bar{z}$ & $\stackrel{\sharp}{\Xi}$ & लొ & ষ্ & $\begin{array}{l}\text { Jे } \\
\text { N }\end{array}$ & $\stackrel{\text { లె }}{\sim}$ & $\stackrel{-}{-}$ & 怘 & $\begin{array}{l}\text { ल् } \\
0\end{array}$ \\
\hline 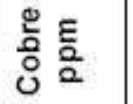 & $\overline{6}$ & $\begin{array}{l}\mathscr{D} \\
\sim \\
\sim\end{array}$ & $\begin{array}{l}\widehat{\infty} \\
\infty \\
\infty\end{array}$ & $\begin{array}{l}80 \\
0\end{array}$ & ฟै & $\begin{array}{l}\text { ?. } \\
\text { ᄋ }\end{array}$ & గొ & ్ֻల & $\frac{m}{6}$ & $\begin{array}{l}\widehat{\emptyset} \\
\infty\end{array}$ & $\begin{array}{l}\text { N } \\
\text { d }\end{array}$ & $\frac{2}{6}$ & $\bar{\sigma}$ & $\begin{array}{l}\mathscr{S} \\
\text { N }\end{array}$ \\
\hline 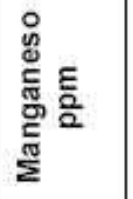 & $\stackrel{m}{-}$ & $\stackrel{0}{\circ}$ & - & $\stackrel{\infty}{=}$ & $\stackrel{0}{-}$ & $\cong$ & $\bar{F}$ & $\stackrel{\infty}{0}$ & $\stackrel{0}{\circ}$ & - & $\stackrel{0}{\longrightarrow}$ & $=$ & $\mp$ & $\cong$ \\
\hline$\frac{ㅇ ㅡ ㄴ ~}{\frac{d}{x}}$ & $\stackrel{\varphi}{\circ}$ & $\stackrel{0}{-}$ & $\hat{\sigma}$ & $\stackrel{ \pm}{=}$ & $\stackrel{N}{N}$ & $\stackrel{\infty}{\circ}$ & ? & $\stackrel{m}{\sim}$ & 0 & $\bar{m}$ & $\begin{array}{l}\text { o } \\
0 \\
0\end{array}$ & 0 & 0 & 0 \\
\hline$\frac{ㅇ ㅡ ㅇ ~}{\frac{0}{0}}$ & 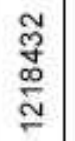 & $\begin{array}{l}\underset{N}{5} \\
\infty \\
\infty \\
\stackrel{\infty}{=}\end{array}$ & $\begin{array}{l}\text { d } \\
\text { d } \\
\text { d. } \\
=\end{array}$ & $\begin{array}{l}\stackrel{2}{\frac{\pi}{8}} \\
\stackrel{8}{\circ}\end{array}$ & 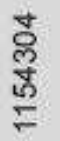 & 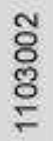 & 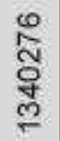 & 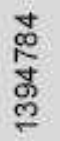 & $\begin{array}{l}\frac{1}{5} \\
\frac{1}{2} \\
\frac{1}{2} \\
0\end{array}$ & 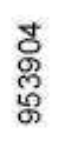 & 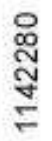 & $\begin{array}{l}\infty \\
0 \\
\varnothing 0 \\
\mathscr{8} \\
0\end{array}$ & $\frac{\text { กै }}{\text { है }}$ & 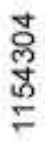 \\
\hline 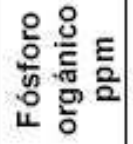 & ळ్ & $\stackrel{m}{E}$ & $E$ & స్లి & $\stackrel{\mathscr{ః}}{\circledR}$ & $\begin{array}{l}\text { :ै } \\
\text { के }\end{array}$ & $\stackrel{\frac{N}{2}}{\frac{N}{N}}$ & స్ & $\stackrel{\Sigma}{\stackrel{n}{\leftarrow}}$ & $\begin{array}{l}\mathscr{D} \\
\mathbb{N}\end{array}$ & $\stackrel{\infty}{\infty}$ & ণ్రి & : & $\frac{m}{\sqrt{v}}$ \\
\hline 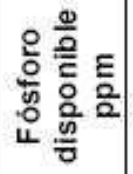 & กี & ભొ & 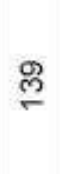 & $\stackrel{2}{\simeq}$ & ถึ & $\stackrel{\text { ㅇ }}{\sim}$ & ఝ్ర & $\underset{\varnothing}{\simeq}$ & $\tilde{\infty}^{\circ}$ & $\stackrel{ \pm}{=}$ & 흥 & 里 & 음 & $\stackrel{f}{+}$ \\
\hline tᄒํㄹ & 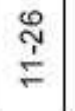 & $\begin{array}{l}0 \\
\text { ?े } \\
\stackrel{\text { dv }}{ }\end{array}$ & $\begin{array}{l}\text { गे } \\
\text { oे }\end{array}$ & $\frac{\circ}{\delta}$ & $\begin{array}{l}\stackrel{2}{~} \\
\text { ô }\end{array}$ & $\begin{array}{l}\text { ? } \\
\text { है } \\
\text { }\end{array}$ & $\begin{array}{l}\text { है } \\
\text { है }\end{array}$ & $\begin{array}{l}\$ \\
\$ \\
15\end{array}$ & $\begin{array}{l}+ \\
0 \\
0 \\
10 \\
0\end{array}$ & 웅 & స̃ & $\begin{array}{l}\text { y } \\
\text { ฝे }\end{array}$ & $\begin{array}{l}\stackrel{0}{0} \\
\stackrel{\sim}{\tilde{y}}\end{array}$ & 怘 \\
\hline 『 & U. & 亗 & $\stackrel{\infty}{\sim}$ & $\varangle$ & 0 & $\stackrel{m}{\sim}$ & ర్ల & बे & ర్ల & $\varangle$ & U⿺辶 & 0 & $\stackrel{\mathscr{N}}{\sim}$ & U్ \\
\hline$\Sigma$ & \multicolumn{3}{|c|}{ Perfil 4} & \multicolumn{6}{|c|}{ Perfil 5} & \multicolumn{5}{|c|}{ Perfil 6} \\
\hline
\end{tabular}

Cuadro 1. Continuación 
Procesos degradatorios intensos que dieron lugar a la formación de barrancos, escarpas de erosión, y cárcavas incipientes y ramificadas son los responsables del estado actual de dicho paisaje.

A pesar de ello pudieron caracterizarse dos tipos de unidades arqueológicas: a) residencial, definido a partir de la fotointerpretación, prospección, artefactos arqueológicos encontrados en el nivel de ocupación prehispánico y la presencia del horizonte superficial (3A); y b) agrícola, determinado por fotointerpretación, prospección y corroborado a través de los análisis de laboratorio.

Si bien el comportamiento de los macronutrientes (fósforo y calcio disponibles) responde a condiciones físico-químicas normales. El de los micronutrientes (hierro, calcio y manganeso disponibles) está supeditado al porcentaje deficiente de materia orgánica que afecta la normal concentración y distribución de aquellos elementos a lo largo del perfil.

Para concluir, se pudo establecer que las estructuras identificadas en el sector pertenecientes a la cultura Tafí fueron utilizadas esencialmente para actividades agrarias y domésticas. Sin embargo, las bajas concentraciones de fósforo orgánico junto con el comportamiento de los micronutrientes producto de los bajos porcentaje de materia orgánica, dan la pauta de la falta de uso de fertilizantes en los suelos sometidos a prácticas agrícolas.

\section{Agradecimientos}

Agradecemos al personal del INGEMA por su apoyo y especialmente al Geól. José Busnelli por la colaboración para el manejo del programa ILWIS 3.4. Las investigaciones realizadas se subvencionaron con fondos de CIUNT, CONICET y ANPCyT.

\section{Bibliografía citada}

Berberián, E. E., A. E. Nielsen, E. Argüello de Dorsch, B. Bixio, L. A. Spalletti, J. A. Salazar, y E. L Pillado.

1988. Sistemas de Asentamiento Prehispánicos del Valle de Tafí. Comechingonia, Córdoba.

Bohn, H. L., B. Macneal, y G. A. O'Connor.

1993. Química del Suelo. Grupo Noriega, México.

Buckman, H. O. y N. C. Brady

1977. Naturaleza y Propiedades de los Suelos. Montaner y Simon, Barcelona.

Butzer, K. W.

1989. Arqueología una ecología del hombre. Bellaterra S.A., Barcelona. 
Collantes, M. M.

2001. Paleogeomorfología y geología del cuaternario de la cuenca del río Tafí, departamento Tafí del Valle, provincia de Tucumán. Tesis Doctoral. Universidad Nacional de Salta, Salta.

Etchevehere, P.

1976. Normas de Reconocimiento de Suelos. 2da ed. Secretaría de Estado de Agricultura y Ganadería de la Nación, Buenos Aires.

González, A. R.

1965. Nuevas fechas de la cronología arqueológica argentina obtenidas por el método de radiocarbón (V). Revista del Instituto de Antropología 2-3: 289-297.

González, A. R. y V. A Nuñez Regueiro

1960. Preliminary report on archaeological research in Tafí del valle, N.W. Argentina. Akten del 34 Internationalen Americanisten Kongresses 34: 485-496.

Nuñez Regueiro, V. A. y J. García Azcárate

1996. Investigaciones arqueológicas en el Mollar, dpto. Tafí del Valle, pcia. de Tucumán. Actas y Memorias XI Congreso Nacional de Arqueología Argentina 13: 87-97. Mendoza.

Riechaman, M. S.

2002. The responses of plants to metals toxicity: A review focusing on cooper, manganese and cinc. Australian Minerals and Energy Environment Foundation. Australia.

Roca, N., M. S. Pazos, y J. Bech

2007. Disponibilidad de cobre, hierro, manganeso, zinc en suelos del NO Argentino. Suelo (Argentina) 25(1):31-42.

Roldán, J., M. M. Sampietro Vattuone y M. A. Vattuone

2005. Técnicas analíticas para el estudio de los suelos de sitios agrícolas prehispánicos. Primer Congreso Argentino de Arqueometría, 60-69. Santa Fe.

Roldán, J., M. M. Sampietro Vattuone, L. del V. Neder, y M. A. Vattuone

2008. Efectos antrópicos del uso del suelo durante el Formativo en el valle de Tafí. Revista Chungara 40(2): 161-172.

Sampietro Vattuone, M. M.

2007. El suelo como artefacto: una experiencia desde el NOA. $2^{\text {do }}$ Congreso Argentino y $1^{\text {ro }}$ Latinoamericano de Arqueometría. Buenos Aires (en prensa).

Sampietro Vattuone, M. M.

2002 Contribución al conocimiento geoarqueológico del valle de Tafí Tucumán (Argentina). Tesis doctoral. Facultad de Ciencias Naturales e I. M. L., Universidad Nacional de Tucumán. San Miguel de Tucumán. 
Sampietro Vattuone, M. M.

1999. Propuesta para un modelo climático del Formativo en el valle de Tafí. XIII Congreso Nacional de Arqueología, 2: 30-31. Brujas, Córdoba.

Sampietro Vattuone, M. M.

1994. Uso del espacio y manejo prehispánico de suelo y agua en el cono del río Blanco. Tafí del valle. Tesis de grado. Facultad de Ciencias Naturales e I. M. L., Universidad Nacional de Tucumán, San Miguel de Tucumán.

Sandor, J. A., P. L. Gersper y J. W. Hawley

1986. Soils at Prehistoric Agricultural Terracing Sities in New Mexico: Phosphorous, Selected Microtutrients, and pH. Soils Science Society American Journal 50: 177-180.

Sayago, J. M. y M. M. Collantes

1991. Evolución paleogeomorfológica del valle de Tafí (Tucumán, Argentina) durante el Cuaternario Superior. Bamberger Geographische Schriften 11:109-124.

Tartusi, M. R. A. y V. A. Nuñez Regueiro

1993. Los centros ceremoniales del NOA. Publicaciones del Instituto de Arqueología 5:1-48.

Terry, R. E., P. J. Hardin, S. D. Houston, S. D. Nelson, J. Jackson, J. Carr, y J. Parnell 2000. Quantitative phosphorus measurement: A field test procedure for archaeological site analysis at Piedras Negras, Guatemala. Geoarchaeology: An International Journal 15:151-16. 